\title{
Technique for Automated Recognition of Sunspots on Full-Disk Solar Images
}

\author{
S. Zharkov \\ Department of Cybernetics, University of Bradford, Bradford, West Yorkshire BD7 1DP, UK \\ Email:s.zharkov@bradford.ac.uk
}

\section{Zharkova}

Department of Cybernetics, University of Bradford, Bradford, West Yorkshire BD7 1DP, UK Email:v.v.zharkova@brad.ac.uk

\section{S. Ipson}

Department of Cybernetics, University of Bradford, Bradford, West Yorkshire BD7 1DP, UK Email: s.s.ipson@bradford.ac.uk

\author{
A. Benkhalil \\ Department of Cybernetics, University of Bradford, Bradford, West Yorkshire BD7 1DP, UK \\ Email:a.k.benkhalil@bradford.ac.uk
}

Received 31 May 2004; Revised 22 February 2005

\begin{abstract}
A new robust technique is presented for automated identification of sunspots on full-disk white-light (WL) solar images obtained from SOHO/MDI instrument and Ca II K1 line images from the Meudon Observatory. Edge-detection methods are applied to find sunspot candidates followed by local thresholding using statistical properties of the region around sunspots. Possible initial oversegmentation of images is remedied with a median filter. The features are smoothed by using morphological closing operations and filled by applying watershed, followed by dilation operator to define regions of interest containing sunspots. A number of physical and geometrical parameters of detected sunspot features are extracted and stored in a relational database along with umbra-penumbra information in the form of pixel run-length data within a bounding rectangle. The detection results reveal very good agreement with the manual synoptic maps and a very high correlation $(96 \%)$ with those produced manually by NOAA Observatory, USA.
\end{abstract}

Keywords and phrases: digital solar image, sunspots, local threshold, edge-detection, morphological operators, sunspot area time series.

\section{INTRODUCTION}

Sunspot identification and characterisation including location, lifetime, contrast, and so forth, are required for a quantitative study of the solar cycle. Sunspot studies also play an essential part in the modelling of the total solar irradiance during the solar cycle. As a component of solar active regions, sunspots and their behaviour are also used in the study of active region evolution and in the forecast of solar flare activity (Steinegger et al. [1]).

This is an open access article distributed under the Creative Commons Attribution License, which permits unrestricted use, distribution, and reproduction in any medium, provided the original work is properly cited.
Manual sunspot catalogues in different formats are produced at various locations all over the world such as the Meudon Observatory, France, the Locarno Solar Observatory, Switzerland, the Mount Wilson Observatory, USA and many others. The Zurich relative sunspot numbers (or since 1981 sunspot index data (SIDC)), compiled from these manual catalogues, are used as a primary indicator of solar activity (Hoyt and Schatten [2,3] and Temmer et al. [4]).

With the substantial increase in the size of solar image data archives, the automated detection and verification of various features of interest is becoming increasingly important for, among other applications, data mining and the reliable forecast of solar activity and space weather. This imposes stringent requirements on the accuracy of the automate 


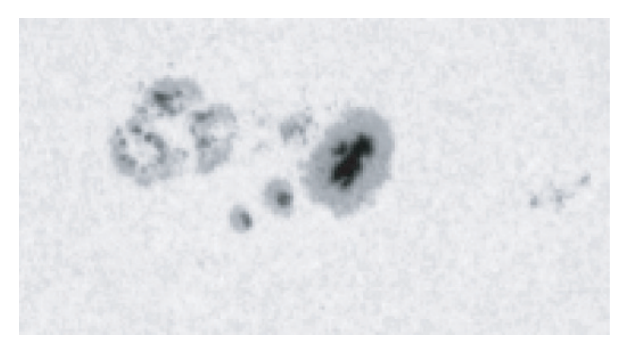

FIGURE 1: A segment of a high-resolution SOHO/MDI image showing sunspots with dark umbras and lighter penumbras on a gray quiet Sun background.

feature detection and verification procedures in comparison with the existing manual ones in order to create a fully automated solar feature catalogue.

A sunspot is a dark cooler part of the Sun's surface. It is cooler than the surrounding atmosphere because of the presence of a strong magnetic field that inhibits the transport of heat via convective motion in the Sun. The magnetic field is formed below the Sun's surface, and extends out into the solar corona. Sunspots are best observed in the visible continuous spectrum also known as "white light" (WL). Larger sunspots can also be observed in Ca II K1 absorption line images as well as in $\mathrm{H} \alpha$ and $\mathrm{Ca}$ II K3 absorption line images. Sunspots generally consist of two parts: a darker, often circular central umbra, and a lighter outer penumbra (see Figure 1).

In white-light and Ca II K1 line digital images sunspots can be characterised by the following two properties: they are considerably darker than the surrounding photosphere and they have well-defined borders, that is, the change in intensity from the quiet photosphere near the sunspot to the sunspot itself occurs over a distance no more than 2-4 arcseconds (or 1-2 pixels for the data used in this study). Most existing techniques for sunspot detection rely on these properties by using thresholding and/or edge-detection operators.

The first thresholding methods for the extraction of sunspot areas used an a priori estimated intensity threshold on white-light full-disk solar images $[5,6,7]$. Sunspots were defined as features with intensity $15 \%$ [6] or $8.5 \%$ $[7,8]$ below the quiet Sun background and sunspot areas were estimated by simply counting all pixels below these values. Similar methods were applied to high-resolution images of solar disk regions containing a sunspot or a group of sunspots using constant intensity boundaries for the umbrapenumbra and the penumbra-photosphere transitions at $59 \%$ and $85 \%$ of the photospheric intensity, respectively $[8,9]$.

For digital solar images the thresholding methods were improved by using image histograms to help determine the threshold levels. Steinegger et al. [1] used the so-called difference histogram method to determine the intensity boundary between the penumbra and the photosphere that was defined for each individual spot. Another method, based on the cumulative sum of sunspot areas contained in the suc- cessive brightness bins of the histogram [10], was applied to determine the umbral areas of sunspots observed in highresolution images capturing a fragment of the solar disk $[10,11]$. A method using sunspot contrast and contiguity, and based on a region growing technique was developed and described by Preminger et al. in [12].

There is also a Bayesian technique for active region and sunspot detection and labelling developed by Turmon et al. [13] that is rather computationally expensive. Moreover, the method is more oriented toward faculae detection and does not detect sunspot umbras. Although the algorithm performs well when trained appropriately, the training process itself can be rather difficult to arrange on images with different background variations corresponding to varying observing conditions.

Another approach to sunspot area measurements utilising edge-detection and boundary gradient intensity was suggested for high-resolution observations of individual sunspot groups, and/or non-full-disk segments by Gyoriri [14]. The method is very accurate when applied to data with sufficiently high resolution. However, in its current form, this method is not suitable for the automated sunspot detection on full-disk images of the low and moderate resolutions that are available in most archives. Therefore, all the existing techniques described above in their original form are not suitable for the automatic detection and identification of sunspots on full-disk images, since their performance depends on the images with high resolution [14] and/or quality [12] that cannot be guaranteed for full-disk images.

In the current paper a new hybrid technique for automatic identification of sunspots on full-disk images using edge-detection is proposed, which is significantly improved by using image standardisation and enhancement procedures. The techniques presented are used for the detection of sunspots on white-light and Ca II K1 line full-disk images, extracting sunspot sizes, locations, umbra and penumbra areas and intensities with high accuracy restricted only by pixel resolution. The techniques can provide fast automated data processing online from ground-based and space-based instruments. The techniques applied for image preprocessing and sunspot detection on white-light and Ca II KI images are described in Section 2, the verification of detected features is presented in Section 3, and the conclusions are drawn in Section 4 .

\section{THE TECHNIQUE FOR SUNSPOT DETECTION}

\subsection{Observations and preprocessing techniques}

\subsubsection{Observations and their synchronisation}

The following two sets of solar full-disk images, provided in the flexible image transport system (FITS) file format (http://fits.gsfc.nasa.gov/), were used for this study: the first was supplied by the Meudon Observatory, and the second was obtained from the MDI instrument aboard the SOHO satellite. Both sets cover the time period spanning April 130,2002 , and July 1-31, 2002, while the SOHO/MDI data were processed for the 8-year period from 1996-2003. 
The Ca II K1 line spectroheliograms from Meudon provide images of the solar photosphere in the blue wing of the Ca II K $3934 \AA$ line, or K1 line taken at a given time $T_{\mathrm{Ca}}$ of solar rotation. These data are acquired once a day on film by performing scans of the solar disk using an entrance slit. The film image is then digitised, providing a pixel size of about 2.3 arcseconds. The other set of data used, from the SOHO/MDI instrument, provides almost continuous observations of the Sun in the white-light continuum in the vicinity of the Ni I $6768 \AA$ line with a pixel size of about 2 arcseconds taken at the time $T_{\mathrm{wl}}$. Intensities of all pixels outside the solar disk in the SOHO/MDI WL images are set to zero.

These images were, in addition, complemented by magnetic field measurements from the line-of-sight (LOS) magnetograms captured by the same SOHO/MDI instrument at the moment $T_{M}$, keeping the data consistent with the WL images. A magnetogram is an image obtained by an instrument, which can detect the strength and location of the magnetic fields from the Zeeman polarisation of the radiation in this field. In the magnetogram shown in Figure 2, gray areas indicate low-magnetic-field regions, while black and white areas indicate regions where there are strong negative and positive magnetic fields, respectively.

For the determination of the magnetic field inside detected sunspot areas the white-light images and magnetograms were synchronised as follows. We rotate a solar magnetogram image to the time, $T_{\mathrm{wl}}$, and point of view corresponding to a WL image using standard IDL solarsoft libraries to allow pixel-by-pixel comparison of both images. Using sunspot detection results, defined on WL images, as masks applied to corresponding synchronised magnetograms allows us to extract pixel values from these areas in magnetic field units calibrated by the SOHO/MDI team (Scherrer et al. [15]).

\subsubsection{Preprocessing technique}

The images from both data sets were preprocessed, with FITS file header information checked and amended where necessary using the techniques described by Zharkova et al. [16]. These techniques include limb fitting; removal of geometrical distortion; centre position and size standardisation.

The limb fitting method has three stages: (1) computing an initial approximation of the disk centre and radius; (2) using edge-detection to provide candidate points for fitting an ellipse using information from the initial estimate; (3) fitting an ellipse to the candidate limb points using a least squares approach to iteratively remove outlying points. The procedure starts by making an initial estimate of the solar centre and radius from image data thresholded at an intensity obtained from an analysis of the image histogram, then smoothes the result by using Gaussian smoothing kernel of size $5 \times 5$ that is recommended by the MDI team (Scherrer et al. [15]) as the first stage of applying Canny edge-detection routine (Canny [17]) to the original 12-bit data. Candidate edge points for the limb are selected using a radial histogram method based on the initial centre estimate and the chosen

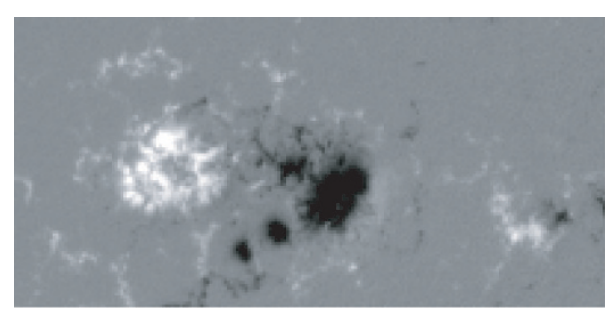

FIGURE 2: A sample magnetogram fragment from SOHO/MDI. The darkest areas are regions of negative magnetic polarity (directed towards the centre of the Sun) and the white areas are regions of positive magnetic polarity (directed towards the observer). The gray areas indicate regions of weak magnetic field.

points fitted to a quadratic function by minimising the algebraic distance using singular value decomposition. The five parameters of the ellipse-fitting the limb are extracted from the quadratic function. These parameters are used to define an affine transformation that converts the image shape into a circle. Transformed images are generated using bilinear interpolation.

Often solar images require intensity renormalisation because of radial limb-darkening [18] caused by the radiation projection from the spherical atmosphere onto a flat solar image that increases the radiation's optical depth towards the limb and results in pixel darkening. This is achieved by fitting a background function to a set of radial sample points having median radial intensities. The median filtering of the radial intensity starts by transformation of a standardised solar disk onto a rectangular image using a Cartesian-to-polar coordinates transformation. The median value of each row is used to replace all the intensities in each row. The median transformation is a very effective way of removing artefacts often present in the images taken from ground-based observatories. However, the presence of nonradial illumination effects in an image such as stripes and lines caused by dust present at the spectral slit may cause larger than sunspot length variations of the background intensity along each row of fixed radius and then the median of the row is no longer an appropriate background estimate but would require a sophisticated segmentation procedure [16]. Such a segmentation procedure is not implemented yet, so these images are automatically disregarded by the software if such nonradial variations are too severe. By removing the limbdarkening, one obtains a "flat," sometimes called "contrast," image [12] of the solar photosphere using the procedure described in the first paragraph of Section 2.1.2 (see Zharkova et al. [16]).

In order to compare the image quality in both data sets, we have used three basic statistical moments of the digital image data values taken from the image headers (SOHO/MDI) [15] or generated from images directly (Meudon). These include mean (formula (1)), variance (formula (2), not plotted here), skewness (the lack of symmetry of pixel values towards the central pixel, formula (3)) and kurtosis (a measure of whether the data are peaked or flat relative to a normal distribution towards the central pixel, formula (4)) which were 
calculated for the full-disk pixel data $x_{j},(j=1, N)$ as follows:

$$
\begin{aligned}
\text { mean } & =\bar{x}=\frac{1}{N} \sum_{j=0}^{N-1} x_{j}, \\
\text { variance } & =\frac{1}{N-1} \sum_{j=0}^{N-1}\left(x_{j}-\bar{x}\right)^{2}, \\
\text { skewness } & =\frac{1}{N} \sum_{j=0}^{N-1}\left(\frac{x_{j}-\bar{x}}{\sqrt{\text { variance }}}\right)^{3}, \\
\text { kurtosis } & =\frac{1}{N} \sum_{j=0}^{N-1}\left(\frac{x_{j}-\bar{x}}{\sqrt{\text { variance }}}\right)^{4}-3 .
\end{aligned}
$$

The results of the comparison between the Ca II K1 and MDI WL data sets for the period February-May 2002 are presented in Figure 3, where (a), (b), and (c) are from the MDI data and (d), (e), and (f) are deduced from the Meudon data. Figures $3 \mathrm{a}$ and $3 \mathrm{~d}$ present the mean, Figures $3 \mathrm{~b}$ and $3 \mathrm{e}$ present the skewness and Figures $3 \mathrm{c}$ and $3 \mathrm{f}$ present the kurtosis calculated for every daily image.

The general quality of Meudon images is highly dependent on atmospheric conditions at the time of the observation. A number of instrumental artefacts which are difficult to eliminate, such as dust lines, are often present in these images, thus making image unsuitable for automated detection. Together, atmospheric conditions and instrumental artefacts produce the variations shown in Figures $3 \mathrm{~d}, 3 \mathrm{e}$, and $3 \mathrm{f}$. The $\mathrm{SOHO}$ satellite data is not subject to clouds as is demonstrated in Figures 3a, 3b, and 3c, though there are dropouts from time to time due to spacecraft problems. Hence, the preprocessing for $\mathrm{SOHO}$ images consisted of limb-darkening removal only, while for Meudon images it included noise filtering with median and/or Gaussian filters. Hence, the preprocessed images containing quiet Sun pixels with darker and, possibly (for the images in Ca II K1 line) brighter features superimposed, which are suitable for sunspot detection.

For a full-disk solar image free of the limb-darkening, the quiet Sun intensity value is established from an image histogram as the intensity with the highest pixel count (see, e.g., Figures $4 \mathrm{a}$ and $4 \mathrm{~b}$ ). Thus, in a manner similar to [1], by analysing the histogram of the flat image, an average quiet Sun intensity, $I_{\mathrm{QSun}}$, can be determined.

\subsection{Description of the technique}

\subsubsection{Automatic detection on the SOHO/MDI white-light images}

The technique developed for the SOHO/MDI data relies on the good quality of the images evident from Figures 3a, $3 \mathrm{~b}$, and $3 \mathrm{c}$. This allows a number of parameters, including threshold values as percentages of the quiet Sun intensity, to be set constant for the whole data set. Since sunspots are characterised by strong magnetic field, the synchronised magnetogram data is then used for sunspot verification by checking the magnetic flux at the identified feature location.

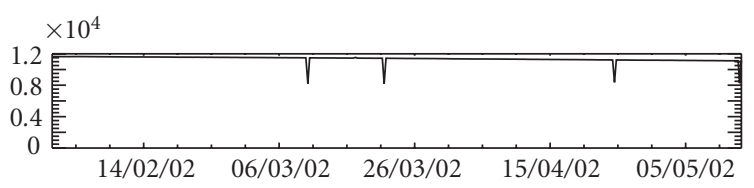

(a)

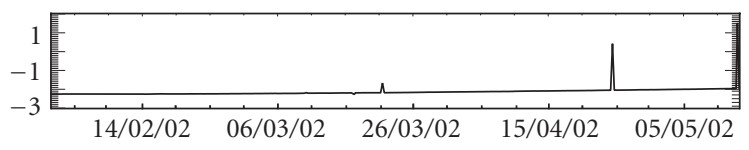

(b)

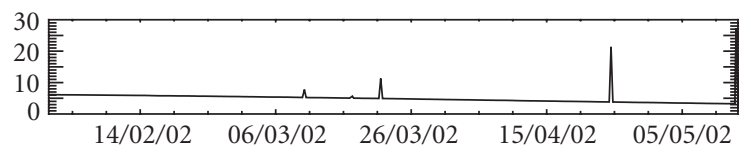

(c)

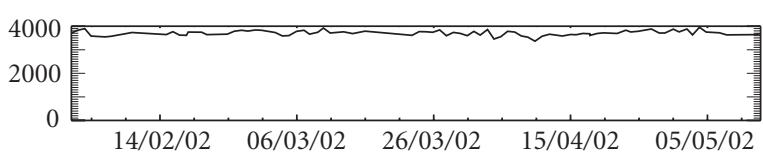

(d)

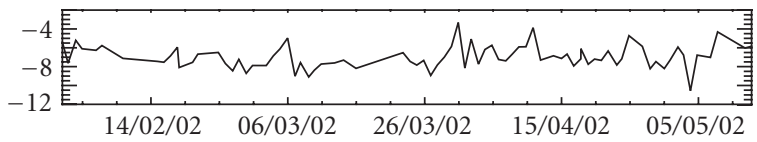

(e)

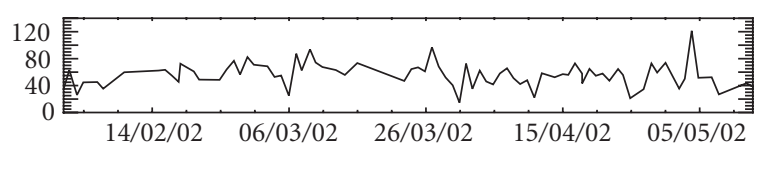

(f)

FIGURE 3: Full-disk data statistics presented for the SOHO/MDI continuum (a) mean, (b) skewness, and (c) kurtosis data sets and for the Meudon Observatory Ca II k1 line (d) mean, (e) skewness, and (f) kurtosis data sets covering February-May 2002. Peaks and dips in the SOHO/MDI plots correspond to defective data (images) that were automatically rejected by our software (the $x$-axis refers to the date of observation and the $y$-axis to the arbitrary intensity units).

Basic (binary) morphological operators such as dilation, closing, and watershed $[19,20]$ are used in our detection code. Binary morphological dilation, also known as Minkowski addition, is defined as

$$
A \oplus B=\left\{x:(\widehat{B})_{x} \cap A \neq \varnothing\right\}=\bigcup_{x \in B} A_{x},
$$

where $A$ is the signal or image being operated on and $B$ is called the "structuring element." This equation simply means that $B$ is moved over $A$ and the intersection of $B$ reflected and translated with $A$ is found. Dilation using disk structuring 


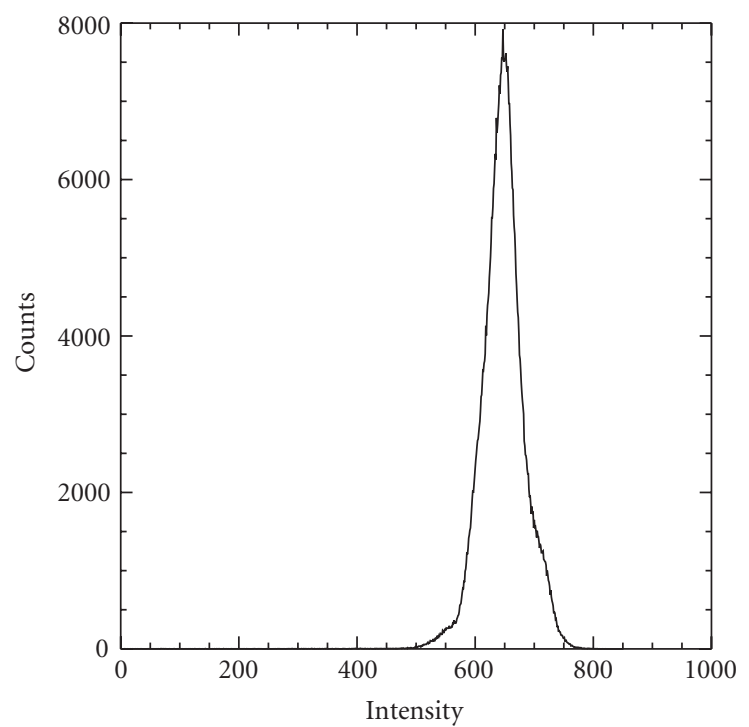

(a)

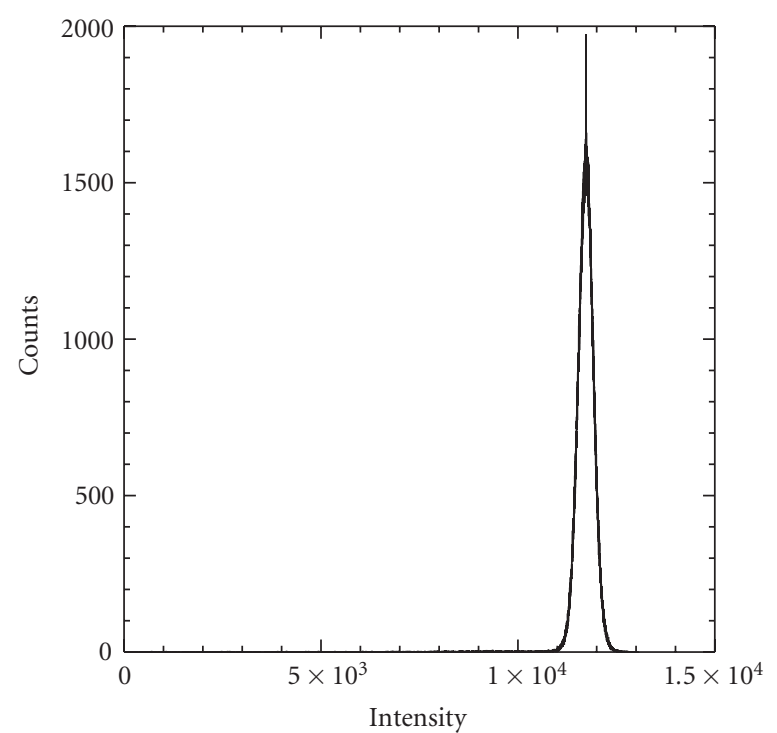

(b)

FIgURE 4: Flat image histograms for the (a) Meudon Observatory Ca II K1 line and (b) SOHO/MDI white-light images taken on April 2 , 2002 and April 1, 2002, respectively.

elements corresponds to isotropic swelling or expansion algorithms common to binary image processing.

Binary morphological erosion, also known as Minkowski subtraction, is defined as

$$
A \Theta B=\left\{x:(B)_{x} \subseteq A \neq \varnothing\right\}=\bigcap_{x \in B} A_{x} .
$$

The equation simply means that erosion of $A$ by $B$ is the set of points $x$ such that $B$ translated by $x$ is contained in $A$. When the structuring element contains the origin, erosion can be seen as a shrinking of the original image.

Morphological closing is defined as dilation followed by erosion. Morphological closing is an idempotent operator. Closing an image with a disk structuring element eliminates small holes, fills gaps on the contours, and fuses narrow breaks and long, thin gulfs.

The morphological watershed operator segments images into watershed regions and their boundaries. Considering the gray scale image as a surface, each local minimum can be thought of as the point to which water falling on the surrounding region drains. The boundaries of the watersheds lie on the tops of the ridges. This operator labels each watershed region with a unique index, and sets the boundaries to zero. We apply the watershed operator provided in the IDL library by Research Systemic Inc. to binary image where it floods enclosed boundaries and thus is used in a filling algorithm. For a detailed discussion of mathematical morphology see the references within the text and numerous books on digital imaging.

The detection code is applied to a "flattened" full-disk SOHO/MDI continuum image, $\Delta$ (Figure $5 \mathrm{a}$ ), with estimated quiet Sun intensity, $I_{\mathrm{QSun}}$ (Figure $4 \mathrm{~b}$ ), image size, solar disk centre pixel coordinates, disk radius, date of observation, and resolution (in arcseconds per pixel). Because of the
Sun's rotation around its axis, a SOHO/MDI magnetogram, $M$, taken at the time $T_{M}$, is synchronised to the continuum image time $T_{\mathrm{WL}}$ via a spatial displacement of the pixels to the position they had at the time $T_{\mathrm{WL}}$ in order to obtain the same point of view as those for the continuum.

The technique presented in the current paper uses edge detection with threshold applied on the gradient image. This technique is significantly less sensitive to noise than the global threshold since it uses the background intensity in the vicinity of a sunspot. We consider sunspots as connected features characterised by strong edges, lower than surrounding quiet Sun intensity, and strong magnetic field. Sunspot properties vary over the solar disk, so a two-stage procedure is adopted. First, sunspot candidate regions are defined. Second, these are analysed on the basis of their local properties to determine sunspot umbra and penumbra regions. This is followed by verification using magnetic information. A detailed description of the procedure is provided in the pseudocode presented in Algorithm 1.

Sunspot candidate regions are determined by combining two approaches: edge-detection and low-intensity-region detection (steps 1-3). First, we obtain a gradient gray-level image, $\Delta^{p}$, from the original preprocessed image, $\Delta$ (Figure 5a) by applying Gaussian smoothing with a sliding window $(5 \times$ 5) followed by Sobel gradient operator (step 1). Then (step 2) we locate strong edges via iterative thresholding of the gradient image starting from initial threshold, $T_{0}$, whose value is not critical but should be small. The threshold is applied followed by $5 \times 5$ median filter and the number of connected components $N_{c}$ and the ratio of the number of edge pixels to the total number of disk pixels $R$ are determined. If the ratio is too large or the number of components is greater than 250, the threshold is incremented. The number 250 is based on the available recorded maximum number of sunspots which 


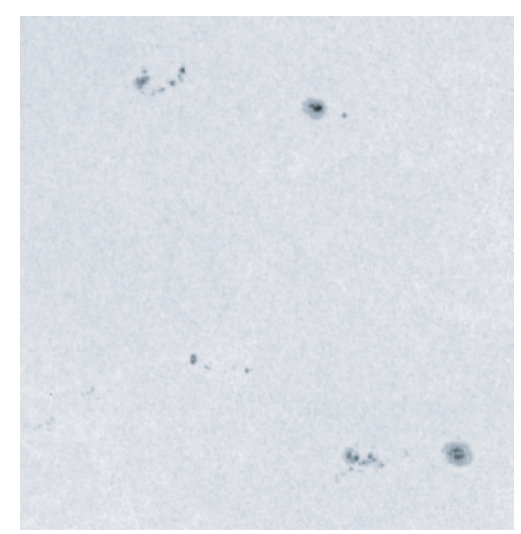

(a)

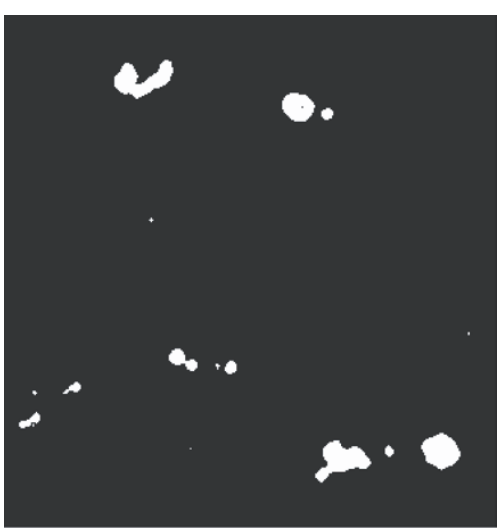

(c)

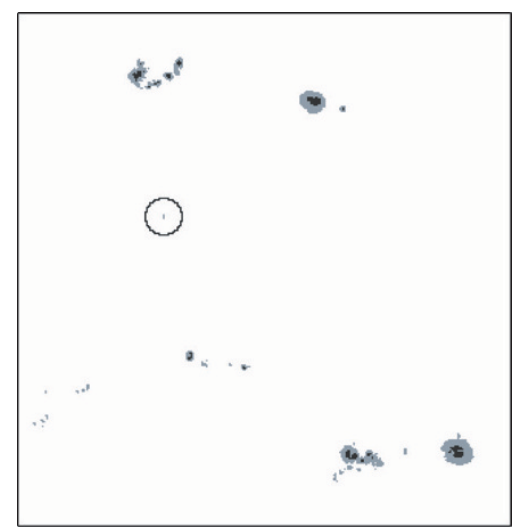

(e)

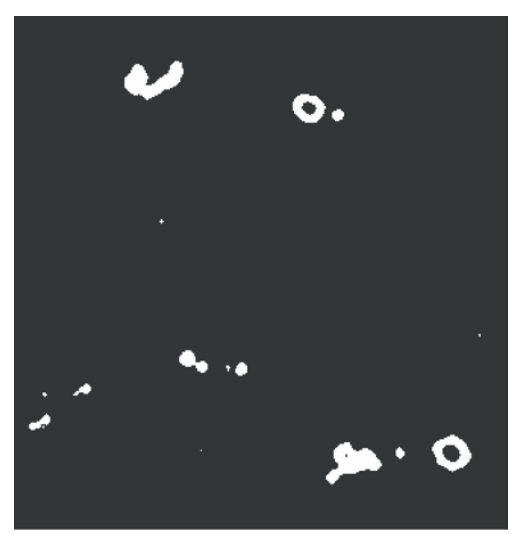

(b)

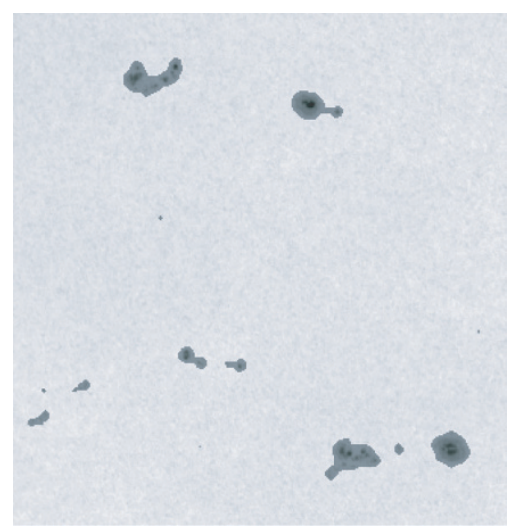

(d)

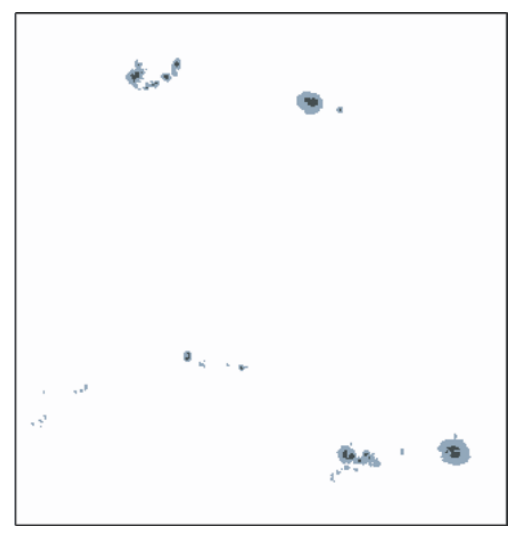

(f)

Figure 5: A sample of the sunspot detection technique applied to the WL disk SOHO/MDI image from April 19, 2002 (presented for a cropped fragment for better resolution): (a) a part of the original image; (b) the detected edges; (c) candidate map; (d) the regions of interest after filtering as masks on original; (e) the detection results before magnetogram verification, false identification is circled; and (f) the final detection results.

is around $170 .{ }^{1}$ Since at this stage of the detection we are dealing with noise and the possibility of several features joined

\footnotetext{
${ }^{1}$ The number varies depending on the observer.
}

into a single candidate region, this limit is increased 250 to ensure that no sunspots are excluded. The presence of noise and fine structures in the original flat image will contribute many low-gradient-value pixels resulting in just a few very large connected regions, if the threshold is too close to zero. 
(1) Apply Gaussian smoothing with sliding window $5 \times 5$ followed by the Sobel operator to a copy of $\Delta$.

(2) Using the initial threshold value, $T_{0}$, threshold the edge map and apply the median $5 \times 5$ filter to the result. Count the number of connected components, $N_{c}$, and the ratio of the number of edge pixels to the total number of disk pixels, $R$ (feature candidates, Figures $5 \mathrm{~b}$ and $6 \mathrm{~b}$ ). If $N_{c}$ is greater than 250 or $R$ is larger than 0.7 , increase $T_{0}$ by set value ( 1 or larger depending on $N_{c}$ and $R$ ) and repeat step 3 from the beginning.

(3) Similarly, iteratively threshold original flat image to define less than 100 dark regions. Combine (using OR operator) two binary images into one binary feature candidate map (Figure 5c).

(4) Remove the edge corresponding to the limb from candidate map and fill the possible gaps in the feature outlines using IDL's morphological closure and watershed operators (Figures $5 \mathrm{~d}$ and $6 \mathrm{c}$ ).

(5) Use blob colouring to define a region of interest, $F_{i}$, as a set of pixels representing a connected component on the resulting binary image, $\bar{B}_{\Delta}$.

(6) Create an empty sunspot candidate map, $B_{\Delta}$, a byte mask which will contain the detection results with pixels belonging to umbra marked as 2, penumbra as 1 .

(7) For every $F_{i}$ extract a cropped image containing $F_{i}$ and define $T_{s}$ and $T_{u}$ :

(i) if $\left|F_{i}\right| \leq 5$ pixels, assign the thresholds: for penumbra $T_{s}=0.91 I_{\mathrm{QSun}}$; for umbra $T_{u}=0.6 I_{\mathrm{QSun}}$,

(ii) if $\left|F_{i}\right|>5$ pixels, assign the thresholds: for penumbra $T_{s}=\max \left\{0.93 I_{\mathrm{QSun}} ;\left(\left\langle F_{i}\right\rangle-0.5^{*} \Delta F_{i}\right)\right\}$; for umbra $T_{u}=\max \left\{0.55 I_{\mathrm{QSun}} ;\left(\left\langle F_{i}\right\rangle-\Delta F_{i}\right)\right\}$, where $\left\langle F_{i}\right\rangle$ is a mean intensity and $\Delta F_{i}$ a standard deviation for $F_{i}$.

(8) Threshold a cropped image at this value to define the candidate umbral and penumbral pixels and insert the results back into $B_{\Delta}$ (Figures $5 \mathrm{e}$ and $6 \mathrm{~d}$ ). Use blob colouring to define a candidate sunspot, $S_{i}$, as a set of pixels representing a connected component in $B_{\Delta}$.

(9) To verify the detection results, cross-check $B_{\Delta}$ with the synchronised magnetogram, $M$, as follows: for every sunspot candidate $S_{i}$ of $B_{\Delta}$ extract

(i) $B_{\max }\left(S_{i}\right)=\max \left(M(p) \mid p \in S_{i}\right)$,

(ii) $B_{\min }\left(S_{i}\right)=\min \left(M(p) \mid p \in S_{i}\right)$,

if $\max \left(\operatorname{abs}\left(B_{\max }\left(S_{i}\right)\right)\right.$, abs $\left.\left(B_{\min }\left(S_{i}\right)\right)\right)<100$, then disregard $S_{i}$ as noise.

(10) For each $S_{i}$ extract and store the following parameters: gravity centre coordinates (Carrington and projective), area, diameter, umbra size, number of umbras detected, maximum-minimum-mean photometric intensity (as related to flattened image), maximum-minimum magnetic flux, total magnetic flux, and total umbral flux.

Algorithm 1: The pseudocode describing the sunspot detection algorithm in SOHO/MDI white-light images.

Imposing an upper limit of 0.7 on the ratio of number of edge pixels to disk pixels excludes this situation. A lower value increment in the iterative thresholding loop ensures better accuracy, at the cost of computational time. The increment value can also be set as a function of the intermediate values of $N_{c}$ and $R$. The resulting binary image (Figure $5 \mathrm{~b}$ ) contains complete and incomplete sunspot boundaries as well as noise and other features such as the solar limb.

Similarly, the original flat image is iteratively thresholded to define dark regions (step 3). The resulting binary image contains fragments of sunspot regions and noise. The two binary images are combined using the logical OR operator (Figure 5c). The image will contain feature boundaries and blobs corresponding to the areas of high gradient and/or low intensity as well as the limb edge. After removing the limb boundary, a $7 \times 7$ morphological closure operator $[19,20]$ is applied to close incomplete boundaries. Closed boundaries are then filled by applying a filling algorithm, based on the IDL watershed function, [21] to the binary image. A $7 \times 7$ dilation operator is then applied to define the regions of interest which possibly contain sunspots (step 4, Figure 5d, regions masked in dark gray).

In the second stage of detection, these regions are uniquely labeled using blob colouring algorithm [22] (step 5) and individually analysed (steps 6-7, Figure 5e). Penumbra and umbra boundaries are determined by thresholding at values $T_{u}$ and $T_{s}$ which are functions of the region's statistical properties and quiet Sun intensity defined in step 7. Practically, the formulae for determining $T_{u}$ and $T_{s}$ (step 7), including the quiet Sun intensity coefficients, 0.91, 0.93, 0.6, 0.55 , are determined by applying the algorithm to a training set of about 200 SOHO/MDI WL images. Since smaller regions of interest (step 7(i)) carry less statistical intensity information, lower $T_{s}$ value reduces the probability of false identification. In order to apply this sunspot detection technique to data sets from other instruments, the values of the constants appearing in the formulas for $T_{u}$ and $T_{s}$ should be determined for each data set. As mentioned in the introduction, other authors [6, 7, 8, 9] apply global thresholds at the different values of 0.85 or 0.925 for $T_{s}$ and 0.59 for $T_{u}$.

In the final stage of the algorithm (steps 8-9), we verify the resulting sunspot candidates by determining the maximum magnetic field within the candidate region (Figure 5f). This information is extracted from synchronised magnetogram $M$, as described in step 9 when we can verify a sunspot candidate as a sunspot, if this magnetic field is higher than the magnetic field threshold. The latter is chosen to be equal to 100 Gauss, that is appropriate for smallest sunspots, or pores [18], and is a factor 5 higher than the noise in magnetic field measurements by the MDI instrument [15].

The method works particularly well for larger features. It also detects a number of smaller features (under 5 pixels) for which there is often not enough information in the continuum image to make a decision whether the detected feature corresponds to a true feature or an artefact (Figure 5e). This detection is verified with great accuracy by using the magnetic field information, extracted from the synchronised magnetograms (Figure 5f). By comparing Figures $5 \mathrm{e}$ and $5 \mathrm{f}$ we can see that the false detection (marked by a circle) between the two top and bottom sunspot groups has been remedied. Detected feature parameters are extracted and stored in the ASCII format in the final step 10. 


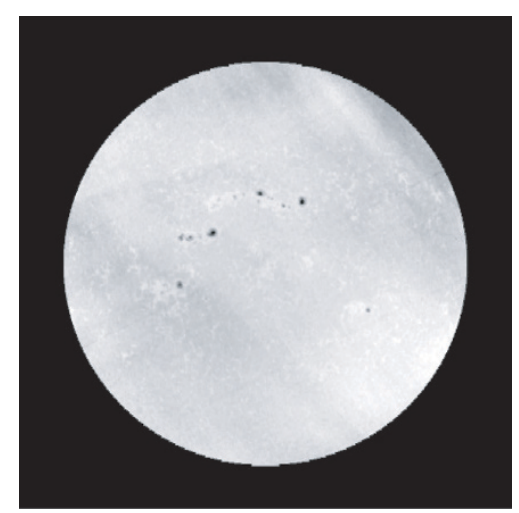

(a)

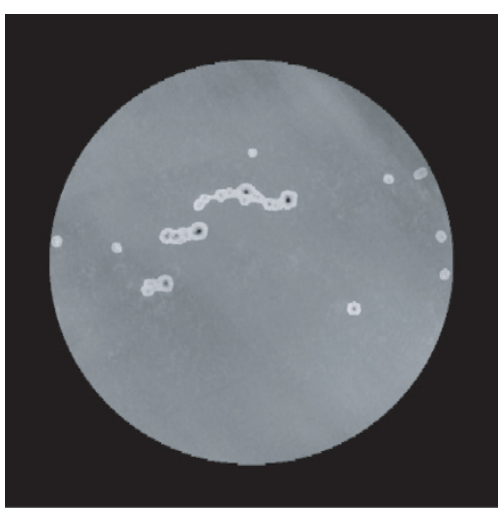

(c)

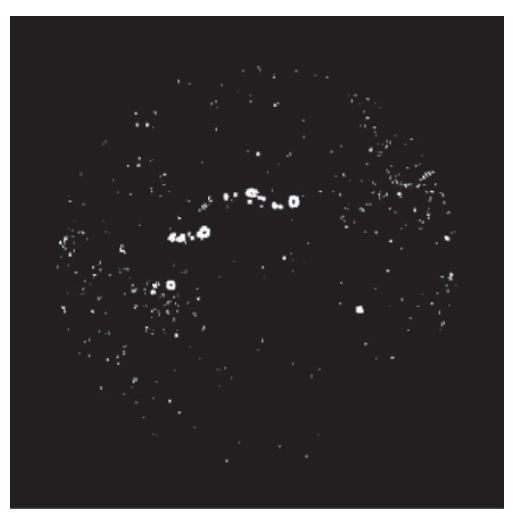

(b)

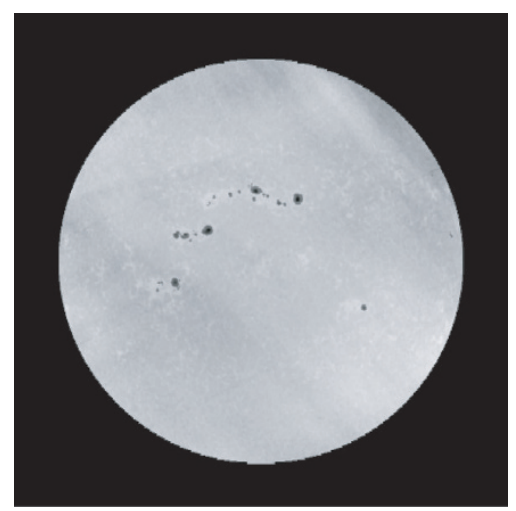

(d)

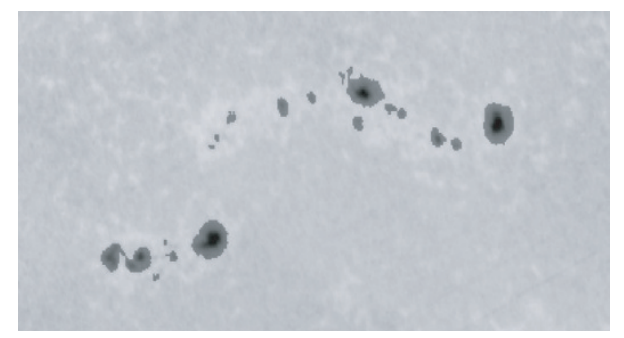

(e)

FIGURE 6: Sunspot detection on the Ca II K1 line full-disk image obtained from Meudon Observatory on April 1, 2002: (a) the original image cleaned; (b) the detected edges; (c) the regions found (dilated); (d) the final detection results superimposed on original image; and (e) the extract of a single sunspot group from (d).

\subsubsection{Technique modifications for Ca II $K 1$ images}

The technique described above can also be applied to Ca II K1 images with the following modifications. First, these images contain substantial noise and distortions owing to instrumental and atmospheric conditions, so their quality is much lower than the SOHO/MDI white-light data (see Figures $3 \mathrm{~d}, 3 \mathrm{e}$, and $3 \mathrm{f}$ ). Hence, the threshold in step 7 (i.e., item 7 in the pseudocode) has to be set lower, that is, $T_{s}=$ $\max \left\{0.91 I_{\mathrm{QSun}} ;\left(\left\langle F_{i}\right\rangle-0.25^{*} \Xi F_{i}\right)\right\}$ for penumbra and $T_{u}=$ $\max \left\{0.55 I_{\mathrm{QSun}} ;\left(\left\langle F_{i}\right\rangle-\Xi F_{i}\right)\right\}$, for umbra, where $\Xi F_{i}$ is the mean absolute deviation of the region of interest $F_{i}$.
The examples of sunspot detection with this technique applied to a Ca II K1 image taken on 2/04/02 are presented in Figure 6. First, the full-disk solar image is preprocessed (Figure 6a) by correcting, if necessary, the shape of the disk to a circular one (via automated limb ellipsefitting) and by removing the limb-darkening as described in Section 2.1.2. Then Sobel edge-detection (similar results were also achieved with morphological gradient operation [19] defined as the result of subtracting an eroded version of the original image from a dilated version of the original image) is applied to the preprocessed image, followed by thresholding in order to detect strong edges (Figure 6b). 
This over segments the image; and then a $5 \times 5$ median filter and an $8 \times 8$ morphological closing filter $[19,20]$ are applied to remove noise, to smooth the edges and to fill in small holes in edges. After removing the limb edge, the watershed transform [21] is applied to the thresholded binary image in order to fill-in closed sunspot boundaries. Regions of interest are defined, similar to the WL case, via morphological closure and dilation $[19,20]$. Candidate sunspot features are then detected by local thresholding using threshold values in the previous paragraph. The candidate features' statistical properties such as size, principal component coefficients and eigenvalues, intensity mean, and mean absolute deviation are then used to aid the removal of false identifications such as the artefacts and lines, often present in the Meudon Observatory images (Figures $6 \mathrm{~d}$ and $6 e)$.

It can be seen that on the Ca II K1 image shown in Figure 6 the technique performs as well as on the white-light data (Figure 5). However, in many other Ca images the technique can still produce a relatively large number of false identifications for smaller features under 10 pixels where there is not enough statistical information to differentiate between the noise and sunspots. This raises the problem of verification of the detected features that is discussed below.

\section{VERIFICATION AND ACCURACY}

There are two possible means of verification. The first option assumes the existence of a tested well-established source of the target data that is used for a straightforward comparison with the automated detection results. In our case, such data would be the records (sunspot drawings) produced by a trained observer. However, the number (and geometry) of visible/detectable sunspots depend on the time (date) of observation (sunspot lifetime can be less than an hour), location of the observer, wavelength, and resolution (in case of digital imaging). Therefore, this method works best when the input data for both detection methods is the same. Otherwise, a number of differences can appear naturally when comparing the two methods.

The second option is comparing two different data sets describing the same sunspots from images taken on the same dates by different instruments, and extracting from each data set a carefully chosen invariant parameter (or set of parameters), such as sunspot area, and looking at its correlation. For our technique, both verification methods were applied and the outcome is presented below.

\subsection{Verification with drawings and synoptic maps}

The verification of the automated sunspot detection results started by comparison with the sunspot database produced manually at the Meudon Observatory and published as synoptic maps in ASCII format. The comparison is shown in Table 1. The two cases presented in Table 1 correspond to two ways of accepting/rejecting detected features. In general, by considering feature size, shape (i.e., principal components), mean intensity, variance, quiet sun intensity, and proximity to other features, one can decide whether the result is likely to be a true feature. In Table 1, case 1, we have included features with sizes over 5 pixels, mean intensities less than the quiet Sun's, mean absolute deviations exceeding 20 (which is about $5 \%$ of the quiet Sun intensity), principal component ratios less than 2.1. In case 2 , we include practically all detected candidate features by setting the deviation and principal component ratio thresholds to 0.05 .

The differences between the manual and automatic methods are expressed by calculating the false acceptance rate (FAR) (where we detect a feature and they do not) and the false rejection rate (FRR) (where they detect a feature and we do not). FAR and FRR were calculated for the available observations for the two different classifier settings described in the previous paragraph. The FAR is lowest for the classifier case 1 and does not exceed $8.8 \%$ of the total sunspot number detected on a day. By contrast, FRR is lowest for the classifier case 2 and does not exceed $15.2 \%$ of the total sunspot number.

The error rates in Table 1 are the consequences of several factors related to the image quality. First, different seeing conditions can adversely influence automated recognition results; for example, a cloud can obstruct a significant portion of the disk, thus greatly reducing the quality of that segment of the image, making it difficult to detect the finer details of that part of the solar photosphere. Second, some small (less than 5-8 pixels) dust lines and image artefacts can be virtually indistinguishable from smaller sunspots leading to false identifications.

Also, in order to interpret the data presented in Table 1, the following points have to be clarified. Sunspot counting methods are different for different observatories. For example, a single large sunspot with one umbra is counted as a single feature at Meudon, but can be counted as 3 or more sunspots (depending on the sunspot group configuration) at the Locarno Observatory. Similarly, there are differences between the Meudon approach and our approach. For example, a large sunspot with several umbras is counted as one feature by us, but can be counted as several features by the Meudon observer. Furthermore, interpretation of sunspot data at Meudon is influenced by the knowledge of earlier data and can sometimes be revised in the light of the subsequent observations. Hence, for instance, on April 2, 2002 there are 20 sunspots detected at Meudon Observatory. The automated detection applied to the same image yielded 18 sunspots corresponding to 17 of the Meudon sunspots with one of the Meudon sunspots detected as two. Thus, in this case FAR is zero, and FRR is 3.

Currently, our automated detection approach is based on extracting all the available information from a single observation and storing this information digitally. Further analysis and classification of the archive data is in progress that will allow us to produce sunspot numbers identical to the existing spot counting techniques.

For the verification of sunspot detection on the SOHO/MDI images, which have better quality (see Figures $3 a, 3 b$, and $3 c$ ), less noise, and better time coverage ( 4 per day), we used the daily sunspot drawings produced manually since 1965 at the Locarno Observatory, Switzerland. 
TABLE 1: The accuracy of sunspot detection and classification for Ca II K1 line observations in comparison with the manual set obtained from Meudon Observatory (see the text for description of defined cases 1 and 2).

\begin{tabular}{cccccccc}
\hline Date & $\begin{array}{c}\text { Number of spots } \\
\text { (Meudon) }\end{array}$ & $\begin{array}{c}\text { Number of spots } \\
\text { (case 1) }\end{array}$ & $\begin{array}{c}\text { FAR } \\
\text { (case 1) }\end{array}$ & $\begin{array}{c}\text { FRR } \\
\text { (case 1) }\end{array}$ & $\begin{array}{c}\text { Number of spots } \\
\text { (case 2) }\end{array}$ & $\begin{array}{c}\text { FAR } \\
\text { (case 2) }\end{array}$ & $\begin{array}{c}\text { FRR } \\
\text { (case 2) }\end{array}$ \\
\hline 01-Apr-02 & 16 & 17 & 2 & 1 & 19 & 4 & 1 \\
02-Apr-02 & 20 & 18 & 0 & 3 & 18 & 0 & 3 \\
03-Apr-02 & 14 & 13 & 0 & 3 & 24 & 10 & 2 \\
04-Apr-02 & 13 & 15 & 2 & 2 & 20 & 6 & 1 \\
05-Apr-02 & 16 & 18 & 0 & 2 & 18 & 1 & 2 \\
06-Apr-02 & 10 & 10 & 0 & 5 & 15 & 5 & 5 \\
07-Apr-02 & 11 & 9 & 1 & 5 & 13 & 4 & 4 \\
08-Apr-02 & 14 & 17 & 3 & 2 & 22 & 7 & 0 \\
09-Apr-02 & 16 & 17 & 0 & 2 & 17 & 0 & 2 \\
10-Apr-02 & 12 & 12 & 0 & 4 & 14 & 1 & 3 \\
11-Apr-02 & 12 & 9 & 0 & 7 & 10 & 1 & 7 \\
12-Apr-02 & 18 & 20 & 2 & 0 & 21 & 3 & 0 \\
14-Apr-02 & 20 & 23 & 2 & 2 & 34 & 13 & 2 \\
15-Apr-02 & 13 & 16 & 1 & 4 & 18 & 2 & 3 \\
16-Apr-02 & 10 & 13 & 3 & 1 & 19 & 9 & 1 \\
17-Apr-02 & 11 & 11 & 1 & 1 & 13 & 2 & 0 \\
18-Apr-02 & 12 & 11 & 1 & 1 & 12 & 1 & 0 \\
19-Apr-02 & 11 & 14 & 0 & 0 & 15 & 1 & 0 \\
20-Apr-02 & 13 & 10 & 0 & 2 & 11 & 1 & 2 \\
21-Apr-02 & 9 & 8 & 1 & 1 & 15 & 7 & 0 \\
22-Apr-02 & 12 & 13 & 1 & 0 & 15 & 3 & 0 \\
23-Apr-02 & 14 & 13 & 0 & 1 & 15 & 1 & 0 \\
24-Apr-02 & 18 & 15 & 0 & 3 & 17 & 0 & 1 \\
25-Apr-02 & 17 & 13 & 0 & 3 & 17 & 2 & 1 \\
27-Apr-02 & 9 & 7 & 0 & 1 & 9 & 2 & 1 \\
28-Apr-02 & 9 & 10 & 1 & 0 & 11 & 2 & 0 \\
29-Apr-02 & 8 & 5 & 0 & 20 & 13 & 0 \\
\hline
\end{tabular}

The Locarno manual drawings are produced in accordance with the technique developed by Waldmeier [23] and the results are stored in the solar index data catalogue (SIDC) at the Royal Belgian Observatory, Brussels [24]. While the Locarno observations are subject to seeing conditions, this is counterbalanced by the higher resolution of live observations (about 1 arcsecond). We have compared the results of our automated detection in white-light images with the available drawings in Locarno for June-July 2002, as well as for January-February 2004 along with a number of random dates in 2002 and 2003 (about 100 daily drawings with up to 100 sunspots per drawing). The comparison has shown a good agreement ( 95\%-98\%). The automated method detects all sunspots visually observable in the SOHO/MDI WL observations. The discrepancies are found at the level of sunspot pores (smaller structures), and can be explained by the time difference between the observations and by the lower resolution of the SOHO/MDI images.

\subsection{Verification with the NOAA data set}

Comparison of temporal variations of the daily sunspot areas extracted from the EGSO solar feature catalogue in 2003 presented in Figure 7b with those available as ASCII files obtained from the drawings of about 365 daily images obtained in 2003 at the US Air Force/NOAA (taken from National Observatory for Astronomy and Astrophysics National Geophysical Data Centre, US [25]), revealed a correlation coefficient of $96 \%$ (Figure 7a). This is a very high accuracy of detection that ensures a good quality of extracted parameters within the resolution limits defined by a particular instrument.

Further verification of sunspot detection in WL images can be obtained by comparing sunspot area statistics with solar activity index such as sunspot numbers. Sunspot numbers are generated manually from sunspot drawings [24] and are related to the number of sunspot groups. The first attempt to compare the sunspot area statistics detected by us with the sunspot numbers revealed a correlation of up to $86 \%$ (see Zharkov and Zharkova [26]). For accurate comparison classification of sunspots into groups is required. Manually this is done using sunspot magnetic field polarity tags and the property that neighbouring sunspots with opposite magnetic field polarities are paired into groups. Implementation of the automated classification of sunspots into groups is the scope of a future paper. 


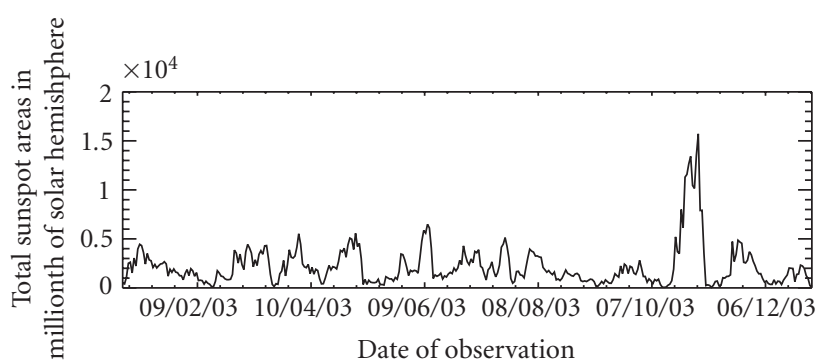

(a)

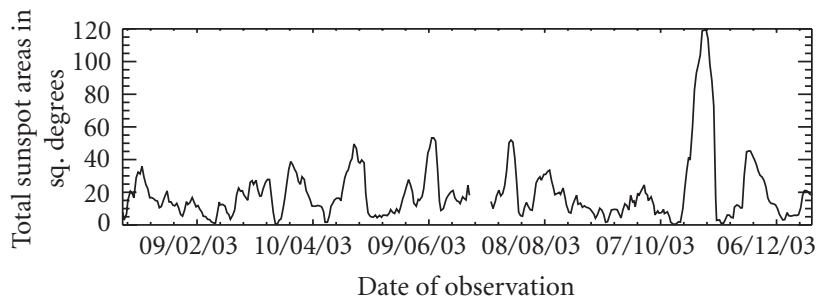

(b)

FIGURE 7: A comparison of temporal variations of daily sunspot areas extracted in 2003 from the (a) USAF/NOAA archive, US, and from the (b) solar feature catalogue with the presented technique.

\section{THE CONCLUSIONS}

New improved techniques are presented for automated sunspot detection on full-disk solar images obtained in the Ca II K1 line from the Meudon Observatory ( $300 \mathrm{im}-$ ages) and in WL from the MDI instrument aboard the SOHO satellite (10082 images).

The technique applies automated image cleaning procedures for elimination of limb-darkening and noncircular image shape. Edge-detection methods and global thresholding methods are used to produce initial image segmentation. The resulting oversegmentation is remedied using a median filter followed by morphological closing operations to close boundaries. A watershed region filling operation, $7 \times 7$ morphological closing and dilation operators are used to define the regions of interest possibly containing sunspots. Each region is then examined separately and the values of thresholds used to define sunspot umbra and penumbra boundaries are determined as a function of the full-disk quiet Sun intensity value and statistical properties of the region (such as mean intensity, standard, deviation, and absolute mean deviation). The sunspots detected in WL are verified using the SOHO/MDI magnetogram data.

The detection results for the selected months in 2002 2003 show a good agreement with the Meudon manual synoptic maps and a very good agreement with the Locarno manual drawings (95\%-98\%). The temporal variations of sunspot areas detected in 2003 with the presented technique from white-light images revealed a very high correlation (96\%) with those produced manually at the National Observatory for Astronomy and Astrophysics (NOAA), US.
A number of physical and geometrical parameters of sunspot features are extracted and stored in the relational database along with run-length encoded umbra and penumbra regions within the bounding rectangle for each sunspot. The database is accessible via web services and (http://solar.inf.brad.ac.uk) website.

In order to significantly reduce the errors contained in acceptance and rejection rate coefficients FARs and FRRs and validate the detection with the existing activity index [25], the sunspot candidate classification into groups has to be implemented. This can be done by examining the adjacent observations, their magnetic polarity, and values, and that is, the scope of a forthcoming paper.

\section{ACKNOWLEDGMENT}

The research has been done for European Grid of Solar Observations (EGSO) funded by the European Commission within the IST fifth framework, Grant IST-2001-32409.

\section{REFERENCES}

[1] M. Steinegger, M. Vazquez, J. A. Bonet, and P. N. Brandt, “On the energy balance of solar active regions," Astrophysical Journal, vol. 461, pp. 478-498, April 1996.

[2] D. V. Hoyt and K. H. Schatten, "Group sunspot numbers: a new solar activity reconstruction," Solar Physics, vol. 179, no. 1, pp. 189-219, 1998.

[3] D. V. Hoyt and K. H. Schatten, "Group sunspot numbers: a new solar activity reconstruction," Solar Physics, vol. 181, no. 2, pp. 491-512, 1998.

[4] M. Temmer, A. Veronig, and A. Hanslmeier, "Hemispheric sunspot numbers $R_{n}$ and $R_{s}$ : Catalogue and N-S asymmetry analysis," Astronomy \& Astrophysics, vol. 390, pp. 707-715, August 2002.

[5] G. A. Chapman and G. Groisman, "A digital analysis of sunspot areas," Solar Physics, vol. 91, pp. 45-50, March 1984.

[6] G. A. Chapman, A. M. Cookson, and J. J. Dobias, "Observations of changes in the bolometric contrast of sunspots," Astrophysical Journal, vol. 432, no. 1, pp. 403-408, 1994.

[7] G. A. Chapman, A. M. Cookson, and D. V. Hoyt, "Solar irradiance from Nimbus-7 compared with ground-based photometry," Solar Physics, vol. 149, no. 2, pp. 249-255, 1994.

[8] M. Steinegger, P. N. Brandt, J. M. Pap, and W. Schmidt, "Sunspot photometry and the total solar irradiance deficit measured in 1980 by ACRIM," Astrophysics and Space Science, vol. 170, no. 1-2, pp. 127-133, 1990.

[9] P. N. Brandt, W. Schmidt, and M. Steinegger, "On the umbrapenumbra area ratio of sunspots," Solar Physics, vol. 129, pp. 191-194, September 1990.

[10] T. Pettauer and P. N. Brandt, "On novel methods to determine areas of sunspots from photoheliograms," Solar Physics, vol. 175, no. 1, pp. 197-203, 1997.

[11] M. Steinegger, J. A. Bonet, and M. Vázquez, "Simulation of seeing influences on the photometric determination of sunspot areas," Solar Physics, vol. 171, no. 2, pp. 303-330, 1997.

[12] D. G. Preminger, S. R. Walton, and G. A. Chapman, "Solar feature identification using contrasts and contiguity," Solar Physics, vol. 202, no. 1, pp. 53-62, 2001.

[13] M. Turmon, J. M. Pap, and S. Mukhtar, "Statistical pattern recognition for labeling solar active regions: application to SOHO/MDI imagery," Astrophysical Journal, vol. 568, no. 1, part 1, pp. 396-407, 2002. 
[14] L. Györi, "Automation of area measurement of sunspots," Solar Physics, vol. 180, no. 1-2, pp. 109-130, 1998.

[15] P. H. Sherrer, R. S. Bogart, R. I. Bush, et al., "The solar oscillations investigation-Michelson Doppler imager," Solar Physics, vol. 162 , no. $1 / 2$, pp. 129-188, 1995.

[16] V. V. Zharkova, S. S. Ipson, S. I. Zharkov, A. Benkhalil, J. Aboudarham, and R. D. Bentley, "A full-disk image standardisation of the synoptic solar observations at the Meudon Observatory," Solar Physics, vol. 214, no. 1, pp. 89-105, 2003.

[17] J. Canny, "A computational approach to edge detection," IEEE Trans. Pattern Anal. Machine Intell., vol. 8, no. 6, pp. 679-698, 1986.

[18] C. W. Allen, Astrophysical Quantities, Athlone Press, London, UK, 1973.

[19] J. Serra, Image Analysis and Mathematical Morphology, vol. 1, Academic Press, London, UK, 1988.

[20] G. Matheron, Random Sets and Integral Geometry, John Wiley \& Sons, New York, NY, USA, 1975.

[21] P. T. Jackway, "Gradient watersheds in morphological scalespace," IEEE Trans. Image Processing, vol. 5, no. 6, pp. 913-921, 1996.

[22] A. Rosenfeld and J. L. Pfaltz, "Sequential operations in digital picture processing," Journal of the Association for Computing Machinery, vol. 13, no. 4, pp. 471-494, 1966.

[23] M. Waldmeier, The Sunspot Activity in the Years 1610-1960, Sunspot Tables, Schulthess Publisher, Zurich, Switzerland, 1961.

[24] Solar Index Data Catalogue, Belgian Royal Observatory, http://sidc.oma.be/products/.

[25] NOAA National Geophysical Data Center, http://www.ngdc. noaa.gov/stp/SOLAR/ftpsunspotregions.html.

[26] S. I. Zharkov and V. V. Zharkova, "Statistical properties of sunspots and their magnetic field detected from full disk SOHO/MDI images in 1996-2003," Adv. Space Res., in press.

S. Zharkov obtained his B.A. (Hons) degree in mathematics from Cambridge University in 1996, his Ph.D. degree from Glasgow University in 2000. He is a postdoctoral Research Assistant at Bradford University, a key developer of the solar feature catalogues for the European Grid of Solar Observations (EGSO) Project. His present interests include digital image processing, solar dynamo theory, and differential geometry.

V. Zharkova obtained an M.S. degree (first class with distinction) in applied mathematics from Kiev State University in 1975, a Ph.D. degree in astrophysics from the Main Astronomical Observatory, National Academy of Sciences of Ukraine in 1984, Advanced Certificate in computing sciences from Kiev State University in 1989. She worked in the Physics and Applied Mathematics Department, Kiev State University,
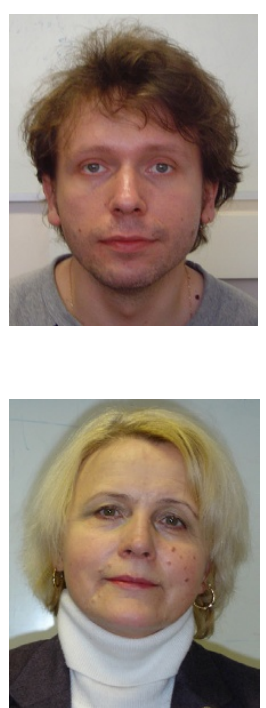
and in the Physics and Astronomy Department, Glasgow University, UK. Currently she is a Professor in applied mathematics at the Department of Cybernetics, Bradford University, UK, and leads the Solar Imaging Research Group. Her current research interests are solar feature recognition from solar images obtained from various space and ground-based observations, flare-induced wave recognition, energy release and transport in solar flares, and prediction of solar activity.
S. Ipson graduated in 1969 with a First Class with Honors degree in applied physics from the University of Bradford, following periods spent at the Rutherford Appleton Laboratory, BP Research Laboratory, Sunbury, and the AEA, Winfrith. After carrying out theoretical and experimental work at AERE, Harwell, and the Universities of Bradford, Oxford, and Heidelberg, he was awarded a Ph.D. degree in theoretical nuclear physics

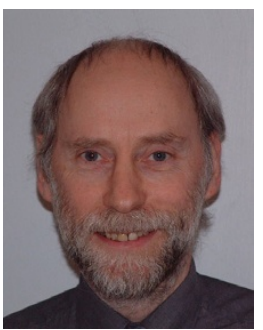
in 1975 from the University of Bradford. He is currently a Senior Lecturer in the Department of Cybernetics, University of Bradford and his present research interests are digital image restoration, pattern recognition, and 3D reconstruction from multiple images.

A. Benkhalil received a B.Eng. degree in computer engineering from The Higher Institute of Electronics, Libya, in 1988, an M.S. degree in real-time electronic systems from University of Bradford, UK, in 1996, and a Ph.D. degree from the same university in 2001 on a project entitled "An FPGAbased real-time autonomous video surveillance system." He is currently a full-time postdoctoral Research Assistant for the Eu-

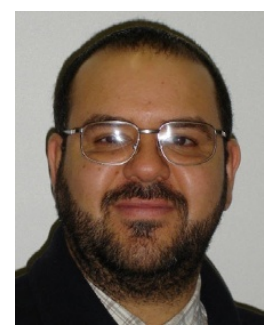
ropean Grid of Solar Observations (EGSO), University of Bradford. His current research interests include image processing, computer vision, and real-time systems design. 